\title{
Spanish Cell Therapy Network (TerCel): 15 years of successful collaborative translational research
}

\author{
Fermín Sánchez-Guijo ${ }^{1}$, Damián García-Olmo ${ }^{2}$, Felipe Prósper ${ }^{3}$, Salvador Martínez ${ }^{4}$, \\ Agustín Zapata ${ }^{5}$, Francisco Fernández-Avilés ${ }^{6}$, Juan José Toledo-Aral ${ }^{7}$, Miguel Torres ${ }^{8}$, \\ Isabel Fariñas ${ }^{9}$, Lina Badimón ${ }^{10}$, José Luis Labandeira-García ${ }^{11}$, Javier García-Sancho ${ }^{12}$, \\ José M. Moraleda ${ }^{13, *}$, on behalf of TerCel \\ ${ }^{1}$ Servicio de Hematología, IBSAL-Hospital Universitario de Salamanca, CIC y CIBERONC, Universidad de Salamanca, Salamanca, Spain \\ 2 “Fundación Jiménez Diaz" University Hospital, Universidad Autónoma de Madrid, Madrid, Spain \\ ${ }^{3}$ Servicio de Hematologia y Terapia Celular, Clinica Universidad de Navarra y CIMA, Universidad de Navarra, Pamplona, Spain \\ ${ }^{4}$ Instituto de Neurociencias UMH-CSIC, CIBERSAM, San Juan de Alicante, Alicante, Spain \\ ${ }^{5}$ Department of Cell Biology, Complutense University, Madrid, Madrid, Spain \\ ${ }^{6}$ Servicio de Cardiología, IiSGM-Hospital General Universitario Gregorio Marañón, Universidad Complutense, CIBERCV, Madrid, Spain \\ ${ }^{7}$ Instituto de Biomedicina de Sevilla (IBiS), Hospital Universitario Virgen del Rocío/CSIC/Universidad de Sevilla, Sevilla, Spain \\ ${ }^{8}$ Cardiovascular Development Program, Centro Nacional de Investigaciones Cardiovasculares, CNIC, Madrid, Spain \\ ${ }^{9}$ Departamento de Biología Celular, Biología Funcional y Antropología Física, Estructura de Recerca Interdisciplinar en Biotecnologia i Biomedicina \\ (ERI BIOTECMED), and CIBERNED, Universidad de Valencia, Valencia, Spain \\ ${ }^{10}$ ICCC Program, IR-Hospital de la Santa Creu I Sant Pau, Autonomous University of Barcelona, Barcelona, Spain \\ ${ }^{11}$ Research Center for Molecular Medicine and Chronic Diseases (CIMUS), University of Santiago de Compostela and Networking Research Center on \\ Neurodegenerative Diseases (CIBERNED), Santiago de Compostela, Spain \\ ${ }^{12}$ Instituto de Biología y Genética Molecular (IBGM), Universidad de Valladolid, Valladolid, Spain \\ ${ }^{13}$ Servicio de Hematología, Hospital Clinico Universitario Virgen de la Arrixaca, IMIB, Universidad de Murcia, Murcia, Spain
}

\section{A R T I C L E I N F O}

\section{Article History:}

Received 30 April 2019

Accepted 1 November 2019

Keywords:

cell therapy

regenerative medicine

research network

stem cells

translational medicine

\begin{abstract}
A B S T R A C T
In the current article we summarize the 15-year experience of the Spanish Cell Therapy Network (TerCel), a successful collaborative public initiative funded by the Spanish government for the support of nationwide translational research in this important area. Thirty-two research groups organized in three programs devoted to cardiovascular, neurodegenerative and immune-inflammatory diseases, respectively, currently form the network. Each program has three working packages focused on basic science, pre-clinical studies and clinical application. TerCel has contributed during this period to boost the translational research in cell therapy in Spain, setting up a network of Good Manufacturing Practice-certified cell manufacturing facilities- and increasing the number of translational research projects, publications, patents and clinical trials of the participating groups, especially those in collaboration. TerCel pays particular attention to the public-private collaboration, which, for instance, has led to the development of the first allogeneic cell therapy product approved by the European Medicines Agency, Darvadstrocel. The current collaborative work is focused on the development of multicenter phase 2 and 3 trials that could translate these therapies to clinical practice for the benefit of patients.

(C) 2019 International Society for Cell and Gene Therapy. Published by Elsevier Inc. This is an open access article under the CC BY-NC-ND license. (http://creativecommons.org/licenses/by-nc-nd/4.0/)
\end{abstract}

\section{Introduction}

In the last two decades, the emerging field of regenerative medicine and cell therapy has drawn the attention of many basic researchers,

\footnotetext{
* Correspondence: Prof. J.M. Moraleda, MD, PhD, Servicio de Hematología, Hospital Clínico Universitario Virgen de la Arrixaca, Ctra. Madrid-Cartagena, El Palmar 30120, Murcia, Spain.

E-mail address: jmoraled@um.es (J.M. Moraleda).
}

clinicians and also the general population. The therapeutic potential of cells for a number of degenerative and/or severe diseases that currently lack a definitive treatment has prompted many private and public initiatives worldwide, especially in the USA and Asian countries [1,2].

The European Union (EU) has the advantage of a common regulatory framework and the existence of the hospital exemption rule for the administration of advanced therapies medicinal products (ATMPs). However, this background has fostered singleinstitution-based experiences and small phase 1 and 2 clinical trials 
Table 1

ATMPs in Europe that obtained MA.

\begin{tabular}{llll}
\hline Product & Disease & Holder of MA & Y \\
\hline ChondroCelect & Cartilage disease & Tigenix & $2009^{\mathrm{a}}$ \\
Glybera & Type 1 hyperlipoproteinemia & UniQure & $2012^{\mathrm{a}}$ \\
MACI & Cartilage disease & Aastrom & $2013^{\mathrm{a}}$ \\
Provenge & Prostate cancer & Dendreon & $2013^{\mathrm{a}}$ \\
Holoclar & Corneal disease & Chiesi & 2015 \\
Imlygic & Melanoma & Amgen & 2015 \\
Strimvelis & Severe combined & GSK & 2016 \\
& $\quad$ inmmunodeficiency & & \\
Zalmoxis & Hematopoietic transplantation & MolMed & 2016 \\
Spherox & Condral lesions & CO.DON & 2017 \\
Alofisel & Perianal fistula in Crohn's disease & Takeda & 2018 \\
Kymriah & Acute lymphoid leukemia and & Novartis & 2018 \\
& $\quad$ diffuse large B-cell lymphoma & & \\
Yescarta & Diffuse large B-cell lymphoma and & Kite & 2018 \\
& primary mediastinal B-cell & & \\
& lymphoma & & \\
\hline
\end{tabular}

EMA, European Medicines Agency, MA, marketing authorization.

a MA was withdrawn or suspended by the EMA afterward due to different reasons.

instead of a coordinated approach leading to large phase 2 and 3 trials, at least at the national level. It is clear that collaborative efforts in this new area of translational research will be needed to achieve the global aim of transferring these treatments from the bench to the bedside within the shortest possible time $[3,4]$. But, as indicated, there are only a small number of published phase 3 trials and very few cell therapy products have reached the final process of authorization by the regulators. As of June 2018, there are only three cell products of 12 ATMPs approved for marketing in Europe (Table 1). It should be noted that approval of ATMP in the EU has a number of inherent difficulties, based on quality issues and the evaluation of post-marketing risk management [5].

In this article we summarize the 15-year experience of the Spanish Cell Therapy Network (TerCel), a successful collaborative public initiative funded by the Instituto de Salud Carlos III (ISCIII), the agency of the Spanish government for the support of nationwide translational research in health sciences.

\section{Description and Objectives of TerCel}

TerCel was established in 2003 with the aim of promoting translational research in cell therapy bringing together both basic research groups and clinical investigators with a common interest in this area. At that time, they were very few experiences in Europe organized through the National Health System research structures to coordinate stem cell research and cellular therapy at the national level. Currently, there are structures similar to TerCel in other EU countries, but each one with their own peculiarities based on the way the translational research has been established in that country. For example, in France they have developed ECell France for Regenerative Medicine (created in 2012), in Italy the Instituti di Ricovero e Cura a Carattere Scientifico (IRCCS) established a number of structures for cell production and regenerative medicine research starting in 2000, the Medical Research Council and other members of the UK Regenerative Medicine Funders Forum had initiatives as the UK Regenerative Medicine Platform (2013) or the Cell Therapy Catapult (2012) and The Netherlands Institute of Regenerative Medicine was established in 2009, among others. It could be of interest, although it is out of the scope of this article, to compare these platforms and structures not only in terms of their characteristics but also of their outcomes.

The work of the TerCel consortium is organized in three main healthcare areas: neurodegenerative diseases (NeuroCel program), cardiovascular diseases (CardioCel program) and inflammatory, immunologic and metabolic diseases (OshiCel program).

Research groups throughout the nation joined the network based on an independent evaluation of their background in terms of scientific output, competitive funding and areas of research. The programs are evaluated yearly and the scientific goals of the different programs have been revisited in 2006, 2012 and 2016. TerCel started with eight groups in 2003, and the number increased to 31 in 2006, when new research groups were incorporated to the network based on their expertise and ability to demonstrate competence and collaboration. Thereafter, the number of research groups has remained stable (between 31 and 33) to date, when 32 research groups are included in the network (10 in CardioCel and 11 in both NeuroCel and OshiCel). The total number of investigators is currently 351, with 31 directly sponsored by TerCel (11 PhD investigators, 12 graduate students and 8 technicians).

There are six major strategic objectives of the network (Figure 1): (i) to understand the mechanisms of repair and the regenerative properties of grafted and endogenous stem cells in tissues and organs, as well as the role of the niche in regulating their function; (ii) to improve stem cell functionality using new technologies such as cell engineering or gene editing; (iii) to develop in vitro and in vivo disease models to get safety and efficacy proof-of-concepts of these improved cell therapies; (iv) to design and improve protocols for the isolation, manipulation, expansion, storage and administration of cells for clinical use under Good Manufacturing Practice (GMP) conditions; (v) to develop collaborative multi-centric clinical trials to translate these therapies to the clinical practice for the benefit of patients; and (vi) to design and launch programs for rigorous dissemination of cell therapy knowledge to society and fight against the fraud in this field.

Each program has a pipeline of three working packages (WPs) aiming at specific objectives, deliverables and milestones to identify cells and ATMPs of benefit in the treatment of human diseases. The first WP of each program (WP1, or basic research WP) is devoted to basic science and mostly includes in vitro analyses and understanding of molecular mechanisms. WP2 (pre-clinical WPs) are focused on pre-clinical studies of stem cell administration in animal models of diseases of the corresponding area. WP3 (clinical WP) involves all the stages of clinical application of cell products, from cell production under GMP conditions to first-in-human clinical trials and advanced collaborative clinical trials.

\section{Management and Regulations of TerCel}

TerCel belongs to a wide program of disease-oriented research networks (RETICs, Thematic Networks for Cooperative Research in Health)
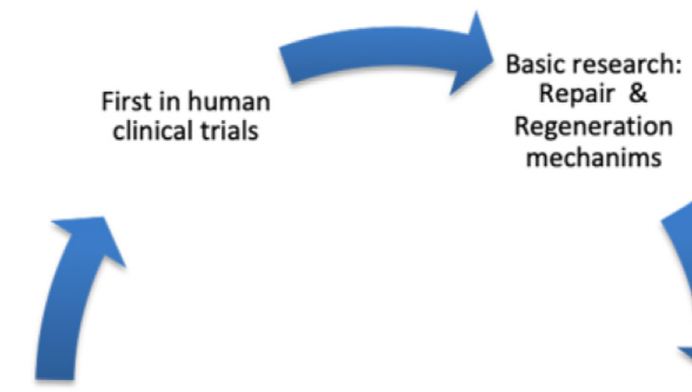

GMP advanced therapies

manufacture.

Clean rooms
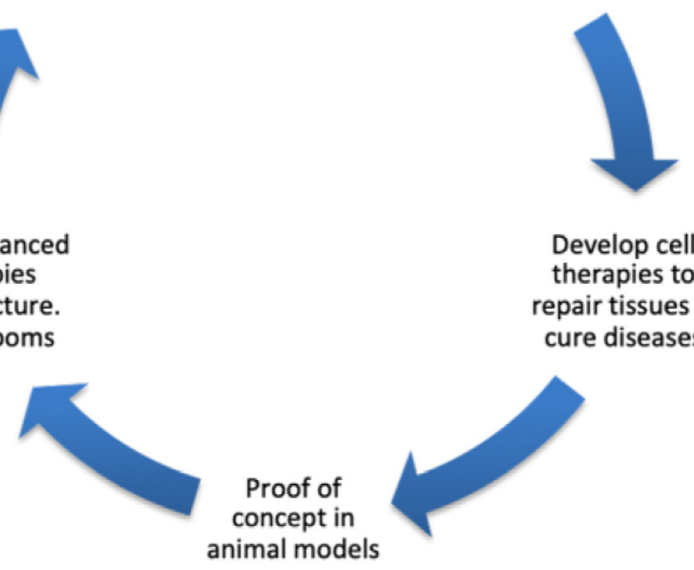

Develop cell therapies to repair tissues \& cure diseases

Fig. 1. Scientific strategy of TerCel. 
funded by the ISCIII. Every 4 years there is a specific competitive call for scientific collaborative proposal applications by the programs of every RETIC, which compete among them for financial support based on a thorough external and independent evaluation.

TerCel is led and coordinated by a Managing Executive Committee (MEC), which is composed of the network general coordinator, the coordinator of each program (CardioCel, NeuroCel and OshiCel) as well as the coordinators of each of the three (basic, pre-clinical and clinical) WPs in each program. The tasks of MEC are as follows: (i) to supervise the advances of the WPs and the accomplishment of deliverables and milestones; (ii) to administer the funding received from the ISCIII; (iii) to foster the scientific collaboration among research groups; (iv) to discuss and design the scientific strategy; (v) to support the investigators in the preparation of scientific meetings and scientific evaluations; (vi) to guarantee the adequate dissemination of the results obtained both to the scientific community and to the general public; and (vii) to establish scientific subcommittees to address specific work priorities. In this last task, TerCel has created several platforms and scientific subcommittees dedicated to the standardization of common protocols for basic research, to establish common animal models of diseases and uniform cell production protocols under GMP conditions, to biobank pluripotent stem cell lines according to the legislation, to facilitate the exchange of investigators between research groups, to interact with the Spanish Medicines Agency (which will be discussed later) and a very important one focused on defining Good Clinical Practices in Cell Therapy. Indeed, the Good Clinical Practices in Cell Therapy committee mission is to clarify all the questions relevant to the patients who are offered cell therapy treatments (there is a specific section in TerCel website devoted to this [6]) and to identify illegal and fraudulent offers of cell therapies in our area of influence. Regarding the GMP facilities platform, each GMP facility shares with the others the academic investigational medical product dossiers and its knowledge for TerCel-initiated collaborative clinical trials, but some of them are also participating in industry-sponsored trials (where some of the manufacturing process could be partially externalized) and in multicenter European clinical trials (where they are either responsible for the production or partners in this process). Funding of the manufacturing by TerCel GMP facilities is always included in the project budget for competitive national and EU grants.

TerCel has also an Internal Scientific Advisory Committee, formed by all principal investigators of the research groups, and an External Scientific Advisory Committee, integrated by four internationally renowned experts in stem cell research and cell therapy. Finally, the General Assembly, formed by all the investigators of the network, meets once a year at the TerCel Annual Scientific Meeting. TerCel also organizes an Annual Technical Workshop meeting open to the public, where internal investigators and/or external experts discuss technology-related advances around a specific topic selected by the MEC each year (e.g., Chimeric Antigen Receptor T cells, organoids, biofabrication). The goal is to analyze in detail emerging concepts in the field and to raise public awareness on the advances at the forefront of cell therapy research.

\section{Main Achievements of TerCel}

When analyzing the period 2013-2017, the number of publications of TerCel investigators add up to 1303 , with a median of 260 manuscripts/year. Of the total number, 209 manuscripts were the result of collaborative efforts of at least two groups of the network, and 140 manuscripts had all authors exclusively from TerCel groups. Therefore, collaboration between groups clearly fostered the generation of synergies that resulted in more and higher impact manuscripts. In this regard, the median impact factor (IF) of all manuscripts published by TerCel members in this period was 4.9 , whereas the IF of the articles published in collaboration between TerCel groups was 6.5. Nevertheless, this is indeed still an IF value that reflects the challenge of publishing translational research studies with a low or medium number of patients in high-impact journals, although the results may be important to further develop the field (as it was during the early years of TerCel). It has to be acknowledged that this fact has further negative consequences for getting additional funding within universities or from other financing structures including the European Commission.

In this same 2013-2017 period, the total number of competitive research projects in which TerCel groups were involved was 487. Seventy of them were the result of collaborations between groups of the network, and in 61 only TerCel members were involved. The total amount of financial support for these TerCel research projects in this period was $144,169,242 €$.

The education program in this period has promoted and financed a median of ten workshops a year related to ATMP therapies and a median of three young investigator exchanges among TerCel groups.

The most important achievements of TerCel in its path from bench to bedside have been the initiation of clinical trials involving ATMPs. Between 2013 and 2017, TerCel groups initiated a total of 111 clinical trials. Of them, 28 were developed in collaboration with TerCel groups and in 22 only groups from the network participated. This is quite an achievement because the public financial support for clinical research in this period was very low due to the country's economic crisis [7]. Before the crisis, Spain was the leading European country in terms of number of funded ATMP clinical trials [8]. This was due to an important financial support from the ISCIII to non-commercial clinical research and to the starting of the TerCel Network in the more salutary period 2004-2010 [8]. More recent research in the database clinicaltrials.gov [9] searching trials on "cell therapy" or "stem cells" between 2011 and 2018 shows that Spain is the second country in Europe in number of clinical trials (68 trials), just after France (95 clinical trials).

Apart from considering all these achievements, with several successful experiences as the development of Darvadstrocel that will be discussed next, there are a number of pitfalls and lessons learned by TerCel throughout this 15-year journey, especially when coordinating all these academic research groups in an over-bureaucratized public system from different regions with sometimes different research structures. Probably the need of early collaboration to develop the preclinical experiments and cooperation in the design of the clinical trials is one of the most important learnings. We have faced scarce knowledge in ATMP trials by Contract Research Organizations when we started, and their cost remains excessive compared with the budget generally obtained to perform clinical trials with ATMPs. Moreover, although public-based Contract Research Organization platforms have been developed in Spain (e.g., CAIBER and later SCReN platform), their work is sometimes limited by scarce budget and personnel. For us, it has been also essential to approach the regulatory agencies in a very early stage of the development of the project. Training in regulatory science in ATMPs is a clear "unmet need" in our scientific and clinical environment. In this regard, we have to stress that the collaboration with the Spanish Medicines Agency (AEMPS) has been crucial to obtain many of TerCel's achievements. In this regard, TerCel investigators have established regular meetings with AEMPS representatives to discuss common problems and areas of interest, and AEMPS has been extremely open to implement the rules to obtain good-quality ATMP products, giving scientific and technical advice in clinical trial application and being rigorous but constructive in the inspections of the GMP facilities, among other interactions with the network.

\section{TerCel and a Case of Successful Public-Private Collaboration in Spain: Darvadstrocel}

Darvadstrocel (named Cx601 during drug development, and now commercially known as Alofisel) is the tenth ATMP approved in the EU 
under this definition (Table 1 ) and the first cell therapy based on allogeneic cells $[10,11]$. This product is based on adipose tissue derived mesenchymal stromal cells (MSCs) and its labeled therapeutic indication is for the treatment of complex perianal fistulas in adult patients with nonactive/mildly active luminal Crohn's disease, when fistulas have shown an inadequate response to at least one conventional or biologic therapy [12]. Its efficacy was demonstrated in a randomized phase 3 clinical trial, in which the primary endpoint was a strict combined remission outcome at week 24 (i.e., clinical assessment of closure of all treated external openings that were draining at baseline, and absence of collections $>2 \mathrm{~cm}$ of the treated perianal fistulas confirmed using masked central magnetic resonance imaging) showed that the experimental arm (Cx601) provided significantly better results than placebo ( $50 \%$ versus $34 \% ; P=0.024$ ), with no differences in secondary effects (anal abscesses and proctalgia) [13]. These results were further confirmed with a longer follow-up [14]. The interesting issue with this product is that the initial idea started back in 2002 in one of the TerCel groups in Madrid (located in the "La Paz" Hospital and Autonomous University of Madrid [UAM]), led by Professor Damian García Olmo. In 2004 they established a fruitful collaboration with the Spanish biotech company Cellerix, which sponsored the first clinical trials with the product. In 2011, the Belgian company Tigenix acquired Cellerix, but the cell production remained based in Tres Cantos (Madrid). Tigenix further developed the product until its approval in March 2018 by the European Medicines Agency after the outcome of the pivotal phase 3 trial ADMIRE-CD [3,14]. Takeda, one of the biggest pharmaceutical companies, with an increased interest in ATMP products, will finally commercialize the drug in Europe and other markets. The model of collaborative work established between the "La Paz" University Hospital, UAM, TerCel network research groups and Tigenix has been the cornerstone for the development of Darvadstrocel. This is an example of how networking and collaborative approaches can propel the transfer of ATMPs based on cell products from academic research groups to patients through biotechnology or big pharmaceutical companies.

\section{TerCel and Future Areas for Improvement}

The Tigenix-La Paz-UAM-TerCel strategy has been a story of success but additional approaches in the same way with other products need to be promoted. TerCel currently holds 13 patents under exploitation (of a total of 63 patents registered by TerCel groups) and more collaboration with biotechnology companies is needed. In addition, the development of large multicenter phase 3 trials within the network should be increased. In this regard, in 2017 three TerCel partners were included in a European multicenter phase $2 \mathrm{~b}$ trial in degenerative disc disease (RESPINE) [15], and in 2018, six phase 2 and 3 multicenter trials have obtained public funding and will be completed in the next few years. These include the ARTROCELL trial (a randomized phase 3 trial comparing intra-articular administration of autologous versus allogeneic MSCs versus hyaluronic acid for knee osteoarthrosis), the NOMA (No More Amputations) trial (another phase 3 trial that compares different doses of MSCs in the treatment of diabetic foot), the AGTPII trial (a phase 2 trial that evaluates the effects of the adipose-derived graft transposition in myocardial postischemic scars), the CaSeC-HF trial (a phase 2 study assessing the safety and efficacy of intracoronary allogeneic cardiac stem cells in ischemic chronic heart failure) and a phase 2 trial that explores the role of intramuscular autologous mononuclear cells in amyotrophic lateral sclerosis. Finally, for the sixth trial (CART19-BE-02), some TerCel groups have started to collaborate in new approaches to cover academic immunotherapy trials with CAR technology [16]. This trial will explore the role of ARI-001 (an academic CAR-T developed at the Hospital Clinic in Barcelona) in patients with CD19+ relapsed or refractory acute lymphoid leukemia.
It is clear that with the GMP facilities network built, the collaborative experience acquired and the partnerships established with some biotechnological and pharmaceutical companies, the current situation should foster the development of new academic-based cell products in more applications. Because for some indications they may co-exist with commercial ATMPs, it will be important to find clinical settings and technological challenges where both strategies may become synergic and avoid competition.

\section{Conclusion}

In summary, the Institute of Health Carlos III approach to promote cell therapy in the Spanish national health system through the TerCel network has proven to be successful. It has provided a framework for scientific interaction and for the promotion of translational research. TerCel has demonstrated that these new therapies can be safely translated to the patients, the feasibility of performing collaborative clinical trials in this field and also the possibility of transferring new products to the market.

\section{Declaration of Competing Interest}

This manuscript has been supported by the Instituto de Salud Carlos III (ISCIII) through the project "RD16/0011/0001: Red de Terapia Celular", from the sub-program RETICS, integrated in the "Plan Estatal de I+D+I 2013-2016" and co-financed by the European Regional Development Fund "A way to make Europe”. D.G.O. has been an Advisory Board member of TiGenix SAU, and received consulting fees from Takeda. He also holds patents 10157355957US and US11/ 167061 related to Darvadstrocel. The remaining authors do not declare any conflict of interest regarding this article.

\section{Author Contributions}

Conception and design, collection and/or assembly of data, manuscript writing: FSG, JMM, DGO, FP, SM, AZ, FFA, JJTA, MT, IF, LB, JLLG, and JGS. All authors have approved the final article.

\section{Acknowledgments}

We are grateful to all TerCel research groups whose principal investigators and members are listed at http://www.red-tercel.com/ cardiocel.asp, http://www.red-tercel.com/neurocel.asp and http:// www.red-tercel.com/oshicel.asp.

\section{References}

[1] Okada K, Sato Y, Sugiyama D, Sawa Y. Establishment of the National Consortium for Regenerative Medicine and National Regenerative Medicine Database in Japan. Clin Ther 2018;40(7):1076-83.

[2] Trounson A. California Institute for Regenerative Medicine: accelerating stem cell therapies in California and beyond. Stem Cells Transl Med 2012;1(1):6-8.

[3] Father John FT. Making access a priority. Ethics has a vital role in fostering collaboration in health care. Health Prog 2007;88(2):67-72.

[4] Richman S, Gee AP, McKenna DH, Traverse JH, Henry TD, Fisk D, et al. Factors affecting the turnaround time for manufacturing, testing, and release of cell therapy products prepared at multiple sites in support of multicenter cardiovascular regenerative medicine protocols: a Cardiovascular Cell Therapy Research Network (CCTRN) study. Transfusion 2012;52(10):2225-33.

[5] de Wilde S, Coppens DGM, Hoekman J, de Bruin ML, Leufkens HGM, Guchelaar HJ, et al. EU decision-making for marketing authorization of advanced therapy medicinal products: a case study. Drug Discov Today 2018;23(7):1328-33.

[6] TerCel R.Information for patients. http://www.red-tercel.com/presentacion.asp; Accesed April 2019

[7] Pain E. European debt crisis. Research cuts will cause ‘exodus' from Spain. Science 2012;336(6078):139-40

[8] Maciulaitis R, D’Apote L, Buchanan A, Pioppo L, Schneider CK. Clinical development of advanced therapy medicinal products in Europe: evidence that regulators must be proactive. Mol Ther 2012;20(3):479-82. 
[9] Clinical Trials Database https://www.clinicaltrials.gov/. Accessed April 2019.

[10] Scott LJ. Darvadstrocel: A Review in Treatment-Refractory Complex Perianal Fistulas in Crohn's Disease. BioDrugs 2018:32:627-34.

[11] Cuende N, Rasko JEJ, Koh MBC, Dominici M, Ikonomou L. Cell, tissue and gene products with marketing authorization in 2018 worldwide. Cytotherapy 2018:20:1401-13.

[12] Product information: Alofisel http://www.ema.europa.eu/documents/productinformation/alofisel-epar-product-information_en.pdf. Accessed April 2019.

[13] Panes J, Garcia-Olmo D, Van Assche G, Colombel JF, Reinisch W, Baumgart DC, et al. Expanded allogeneic adipose-derived mesenchymal stem cells (Cx601) for complex perianal fistulas in Crohn's disease: a phase 3 randomised, double-blind controlled trial. Lancet 2016;388(10051):1281-90.

[14] Panes J, Garcia-Olmo D, Van Assche G, Colombel JF, Reinisch W, Baumgart DC, et al. Long-term Efficacy and Safety of Stem Cell Therapy (Cx601) for Complex Perianal Fistulas in Patients With Crohn's Disease. Gastroenterology 2018;154(5). 1334-1342 e1334.

[15] RESPINE. https://cordis.europa.eu/project/rcn/207207_es.html. Accesed April 2019

[16] de Wilde S, Guchelaar HJ, Zandvliet ML, Meij P. Understanding clinical development of chimeric antigen receptor T cell therapies. Cytotherapy 2017;19(6):703-9. 